\title{
A Rare Case Presented with Asthma Symptoms: Scimitar Syndrome
}

\author{
Hatice Çelik Tuğlu ${ }^{1}$, Önder Öztürk ${ }^{1, *}$, Merve Ergün ${ }^{2}$, Mustafa Karabacak ${ }^{3}$, \\ Veysel Atilla Ayyıldız ${ }^{4}$, Ahmet Akkaya ${ }^{1}$ \\ ${ }^{1}$ Department of Chest Diseases, Medical School of Süleyman Demirel University, Isparta, Turkey \\ ${ }^{2}$ Medical School of Süleyman Demirel University, Isparta, Turkey \\ ${ }^{3}$ Department of Cardiology, Medical School of Süleyman Demirel University, Isparta, Turkey \\ ${ }^{4}$ Department of Radiology, Medical School of Süleyman Demirel University, Isparta, Turkey \\ *Corresponding author: onderozturk@sdu.edu.tr
}

Received July 18, 2019; Revised August 20, 2019; Accepted August 28, 2019

\begin{abstract}
Background: Scimitar syndrome is a rare constellation of congenital conditions pertaining to partial anomalous pulmonary venous return to the inferior vena cava, which may associate with variable right lung hypoplasia, right pulmonary artery hypoplasia, pulmonary sequestration together with the presence of aortopulmonary collaterals from the descending aorta towards the right lung. Depending on the severity of the defect, it can present as early as in neonatal period or occasionally later in life. In many cases, there are also other associated cardiac anomalies. Case characteristics: 23-year-old woman with recurrent episodes of breathlessness and wheezing symptoms since her childhood. Computed tomography angiogram revealed right pulmonary veins draining into inferior vena cava through a single vein with a single ostium diagnosed as Scimitar syndrome. Outcome: Successfully managed with surgical correction. Message: Scimitar syndrome should be considered in adulthood with asthma symptoms if it is not under control.
\end{abstract}

Keywords: scimitar syndrome, dyspnea, asthma, pulmonary vein anomalies

Cite This Article: Hatice Çelik Tuğlu, Önder Öztürk, Merve Ergün, Mustafa Karabacak, Veysel Atilla Ayyıldız, and Ahmet Akkaya, "A Rare Case Presented with Asthma Symptoms: Scimitar Syndrome.” American Journal of Medical Case Reports, vol. 7, no. 11 (2019): 280-283. doi: 10.12691/ajmcr-7-11-5.

\section{Introduction}

Scimitar syndrome is one of a multitude of rare congenital pulmonary venolobar syndromes that is named for its characteristic radiographic finding - the shadow of an abnormal pulmonary vein which resembles a Turkish sword called a scimitar [1]. It is further associated with hypoplasia of the right lung, congenital heart malformation, an anomalous systemic arterial supply to the lung. Most often, the anomalous pulmonary vein drains into the inferior vena cava (IVC), but there are also case reports of the hepatic vein drainage into the right atrium and the left atrium [2]. Although the exact cause or any genetic mutation have not been identified, females are more frequently affected than males. Its incidence is reported to be $1-3$ per $100000[3,4,5,6]$.

We report a case of an adult female presenting with acute dyspnea and wheezing, who was later diagnosed with scimitar syndrome because of anomalous pulmonary venous return to the IVC.

\section{Case Presentation}

A 23-year-old woman with wheezing symptom since her childhood and increased shortness of breath was referred to our hospital. She had allergic complaints since her childhood and her skin prick test was positive for Dermatophagoides mix, molds, and cotton. She never used cigarettes and was not exposed to asbestos and biomass. Her family history was normal. During physical examination, oxygen saturation was measured 98\% by portable finger pulse oximeter, but no rales and rhonchi were heard. Although she was using budesonide/formoterol inhaler and leukotriene antagonists, her shortness of breath continued, but in modified form. Her pulmonary function test showed FVC 74\% (2,63 L), FEV1 57,4\% (1,78 L), FEV1/FVC 66,96\%, and a reversibility test was positive. Laboratory tests on blood were normal, but total Ig $\mathrm{E}$ was found elevated (208 IU/mL). The chest X-ray showed enlarged pulmonary hilum, increased area of the pulmonary artery trunk, and enlargement of the right atrium contour (Figure 1).

Electrocardiogram showed ST depression and $\mathrm{T}$ negativity in V1-4 and SR pattern with right bundle branch block (incomplete) (Figure 2a and b), Transthoracic echocardiography showed the enlarged right atrium and right ventricle, ventricular septal defect (VSD) with a partial flow through the tricuspid septal valve, tricuspid regurgitation, and ejection fraction of $60 \%$. Pulmonary artery pressure was $35 \mathrm{mmHg}$. Contrastenhanced Thorax CT showed atresia of the upper lobe of the right lung with decreased right lung volume. The right 
pulmonary veins opened to inferior vena cava through a single vein with a single ostium (Figure $3 a$ and $b$ ). The diagnosis of the adult form of scimitar syndrome presenting with mild PAH was made based on the CT angiogram findings. $3 \mathrm{D}$ volume rendered CT scan image shows the aberrant vein draining and entering to the right atrium (Figure 4). She consulted with a cardiologist and was offered intracardiac repair. She was sent to cardiothoracic surgery and successfully managed with surgical correction.

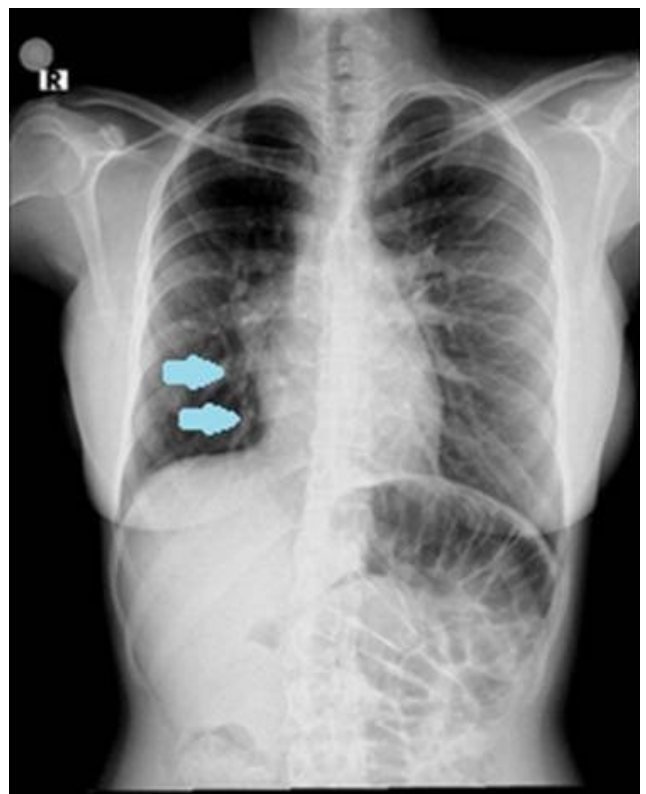

Figure 1. Chest X-ray: Enlarged pulmonary hilum, increased area of the pulmonary artery trunk, and enlargement of the right atrium contour (blue arrows)

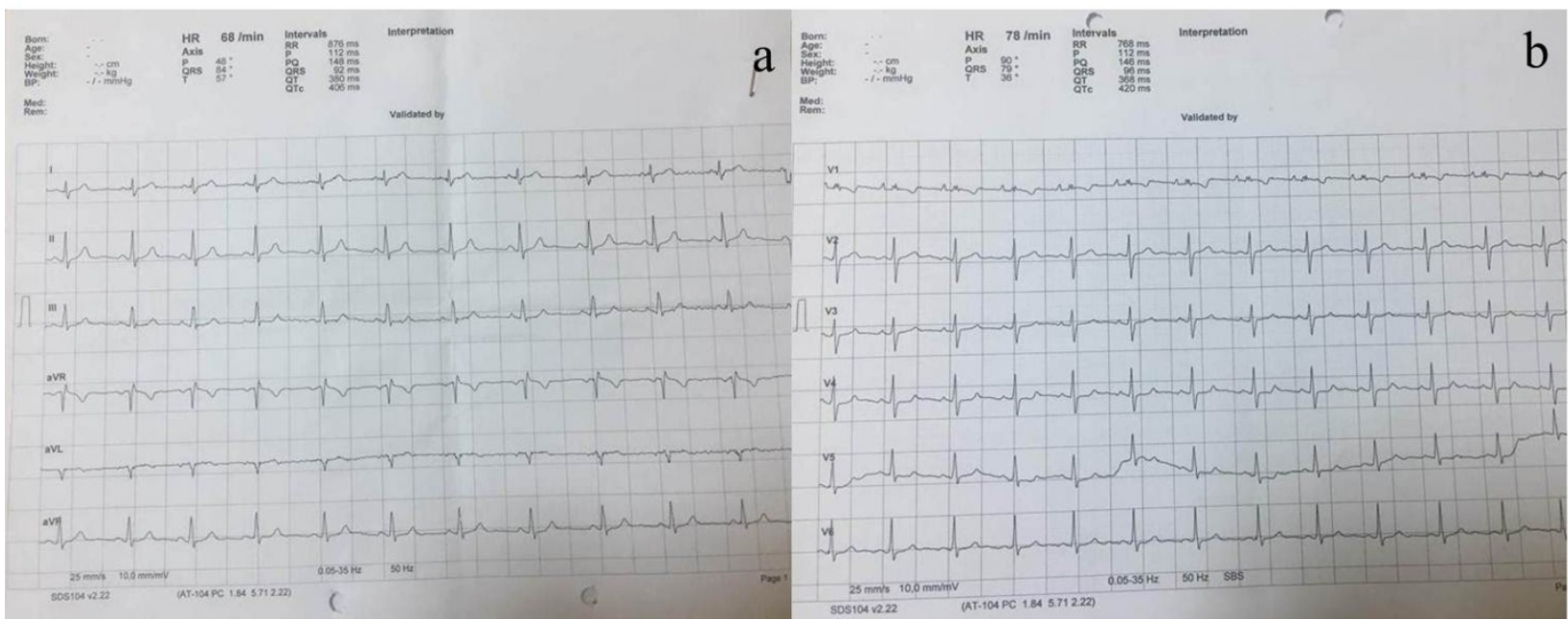

Figure 2. (a) ECG: ST depression and T negativity in V1-4 and (b) SR pattern with right bundle branch block (incomplete)
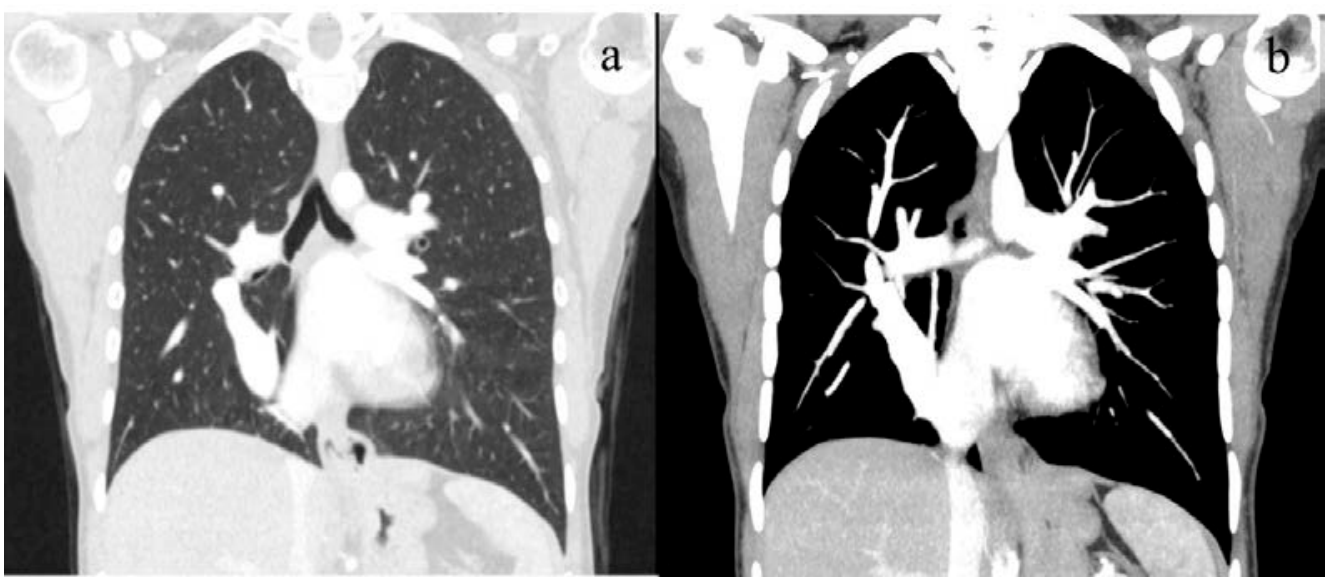

Figure 3. (a) Computed tomography: showed atresia of the upper lobe of the right lung with decreased right lung volume. (b) The right pulmonary veins opened to inferior vena cava through a single vein with a single ostium. 


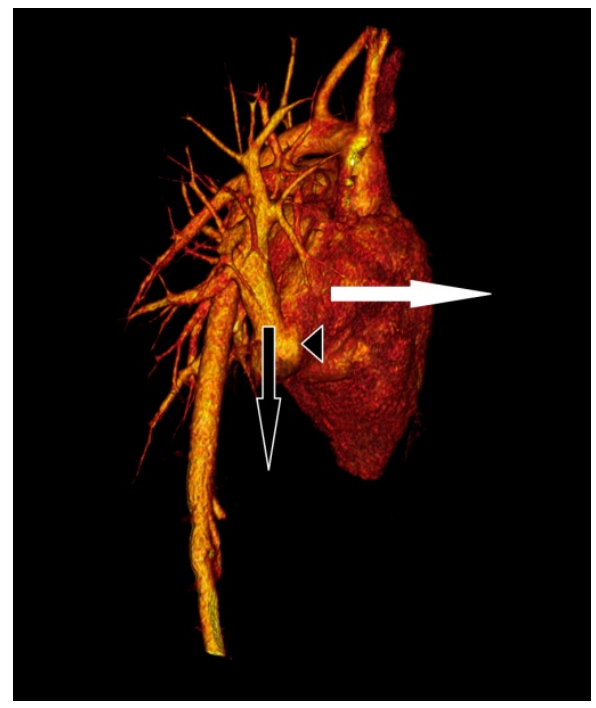

Figure 4. 3 D volume rendered CT scan image: black arrow; aberrant vein draining to the atrium, black arrowhead; entry level of aberrant vein to the right atrium, white arrow; right atrium

\section{Discussion}

Scimitar syndrome is a rare congenital disorder which is seen more often in females than males with an incidence of $1-3$ per $100000[4,5,6]$. Although scimitar syndrome can present with a wide clinical spectrum of symptoms either early in the neonatal period or later in life, the pathogenesis is not known, but it is considered that it may be related to lung bud formation during embryogenesis [7]. Therefore, Dupuis et al., divided scimitar syndrome into 3 main forms: an infantile form with symptoms and pulmonary hypertension, an "older" adult form distinguished by being asymptomatic in infancy, and a form with associated congenital cardiac anomalies. The most common associated anomalies include hypoplasia of the right lung, dextroposition of the heart, hypoplasia of the right pulmonary artery, anomalous systemic arterial supply from the aorta to the right lung, pulmonary sequestration, ventricular septal defect (VSD), and atrial septal defect (ASD) $[1,4,8,9,10]$. The minor or rare components of CPVS include tracheal trifurcation, eventration of the diaphragm, partial absence of the diaphragm, phrenic cyst, horseshoe lung, esophageal and gastric lung, anomalous superior vena cava, and absence of the left pericardium $[11,12,13,14]$. According to Dupius classification, we can classify our case as an "older" adult form distinguished by being asymptomatic in infancy. About $70 \%$ of patients with scimitar syndrome have an associated ASD [15]; in our case, we diagnosed ventricular septal defect, pulmonary arterial hypertension with hypoplasia of the right lung.

However, many of these patients are incidentally diagnosed during infancy and adulthood and usually remain asymptomatic or mildly symptomatic for many years, leading a normal life without surgical repair. Therefore, the clinical presentation of this syndrome is variable depending on the underlying cause. Infants can present with severe defects, such as respiratory problems, feeding difficulty, cyanosis, and heart failure. The majority of older children who are diagnosed after the age of one remain asymptomatic, and adults are also diagnosed when characteristic chest radiographic findings are collected incidentally [16]. Recurrent pneumonia might be another problem that arise from extrinsic compression of an airway from the scimitar vein, although this may not be a unifying mechanism [17]. In our case, the patient was suffering from dyspnea and wheezing since her childhood and initially was diagnosed with allergic rhinitis and asthma, therefore, scimitar syndrome was diagnosed incidentally on chest radiograph, and later, by thorax CT angiogram. In our case, the right pulmonary veins opened to inferior vena cava through a single vein with a single ostium with the atresia of the upper lobe with decreased right lung volume. Another clinical presentation of scimitar syndrome is pulmonary artery hypertension (PAH). Pulmonary artery hypertension is seen mostly in infants with associated congenital heart malformations or with an anomalous large systemic arterial supply to the right lung [18]. A less common cause of pulmonary artery hypertension is the presence of scimitar vein stenosis. In our case, the findings in thorax CT angiogram were associated with pulmonary artery hypertension which was confirmed by measuring the pulmonary artery pressure (35 mmHg) by echocardiography. In agreement with previous reports, the presence of $\mathrm{PAH}$ is the most important prognostic factor in a patient outcome and may be related to the presence of coexisting CHDs [9], in our case, the enlargement of the right atrium and ventricle was shown in an echocardiogram which confirmed CHD.

Adult patients typically remain asymptomatic, but some adult patients may present with symptoms of fatigue, dyspnea, or recurrent pneumonia, however, most patients with isolated scimitar syndrome have an excellent prognosis [9]. Severe cardiac failure, or severe pulmonary hypertension associated with scimitar syndrome might be the main factors for poor prognosis. Although our patient was referred to our hospital with dyspnea and wheezing symptoms due to asthma and allergic rhinitis, the dyspnea symptoms were never improved by the bronchodilator therapy. While we were investigating the etiology of dyspnea and radiologic findings, we found hypoplasia of the right lung, ventricular septal defect, and pulmonary hypertension without cardiac failure in our patient. 
Many patients are incidentally diagnosed during infancy and adulthood and usually remain asymptomatic or mildly symptomatic for many years, leading a normal life without surgical repair. Long-term follow-up observations are recommended for these patients if they have small left-to-right cardiac shunt. However, surgery has been the standard therapy for symptomatic patients who have pulmonary hypertension or poor hemodynamic measurements (a ratio of pulmonary to systemic blood flow greater than 2:1) of left-to-right cardiac shunt. The surgical repair of scimitar syndrome consists of redirecting the pulmonary venous drainage into the left atrium (LA), either baffling the anomalous drainage into the LA via a tunnel or transecting the "scimitar drainage" near its entrance into the IVC, and then reimplanting it directly into the LA. In cases with associated pulmonary sequestration and recurrent pneumonia, a lobectomy or pneumonectomy may be considered $[19,20]$. In our patient, the condition was fully explained to the family and she was sent to cardiac surgery because of being symptomatic and having pulmonary hypertension.

\section{Conclusion}

Scimitar syndrome is a rare congenital heart disease which may vary in clinical presentation, from asymptomatic patients to patients with heart failure associated with pulmonary hypertension. Although the majority of patients are asymptomatic, dyspnea may be the main symptom for hospitalization. Therefore, a complete investigation is necessary in order to determine the etiology of dyspnea. Although scimitar syndrome is usually diagnosed incidentally, physicians should consider the possibility of scimitar syndrome if there is a suspicious lesion on radiographs. Furthermore, continuous clinical and noninvasive further observations are recommended for these patients to determine any clinical or physiological variations and to plan the most appropriate treatment. Nonetheless, when scimitar syndrome is associated with other CHDs and $\mathrm{PAH}$, there is an increased risk of CHF and mortality, and a surgical correction might be considered for these patients. As a result, we would like to present our case in order to draw attention to scimitar syndrome and to take it into account for determination of the etiology of dyspnea.

\section{Conflicts of Interest}

The authors report no conflict of interest.

\section{Financial Disclosure}

There was no financial support or funding for this case report.

Compliance with Ethical Standards and Informed Consent: Informed consent was obtained for publication of this case report.

\section{References}

[1] Dupuis, C., Charaf, L.A., Brevière, G.M., Abou, P., Rémy-Jardin, M., Helmius, G. The "adult" form of the scimitar syndrome, Am. J. Cardiol. 1992; 70(4): 502-507.

[2] Jackson, N., Nokes. B.T., Sakata, K., Cummings, K., Vaszar, L. An unusual variant of scimitar syndrome predisposing to recurrent pneumonia. Respir Med Case Rep. 2019; 26: 240-243.

[3] Khan, S.A., Ahmad, Z., Scimitar syndrome: A rare case of recurrent pneumonia. J Pak Med Assoc. 2019; 69(3): 423-425.

[4] Midyat, L., Demir, E., Aşkin, M., Gülen, F., Ulger, Z., Tanaç, R., Bayraktaroğlu, S. Eponym. Scimitar syndrome. Eur J Pediatr. 2010; 169(10): 1171-1177.

[5] Juraszek, A.L., Cohn, H., Van Praagh, R., Van Praagh, S. Isolated left-sided scimitar vein connecting all left pulmonary veins to the right inferior vena cava. Pediatr Cardiol. 2005; 26(06): 846-847.

[6] Fraser, R.S., Müller, N.L., Colman, N., Parè, P.D. Diagnosis of Diseases of the Chest. 4th ed. Philadelphia: WB Saunders Company, 1999, 653-655.

[7] Clements, B.S, Warner, J.O. Pulmonary sequestration and related congenital bronchopulmonary vascular malformations: no men clature and class ification based on anatomical and embryological considerations. Thorax 1987; 42: 401-408.

[8] Neill, C.A., Ferenca, C., Sabiston, D.C. The familial occurrence of hypoplastic right lung with systemic arterial supply and venous return, "scimitar syndrome” Bull Johns Hopkins Hosp. 1960; 107: $1-21$.

[9] Vida, V.L., Padrini, M., Boccuzzo. G., Agnoletti, G., Bondanza, S., Butera, G., Chiappa, E., Marasini, M., Pilati, M., Pongiglione, G., Prandstraller, D., Russo, M.G., Castaldi, B., Santoro, G., Spadoni, I., Stellin, G., Milanesi, O; Italian Society of Pediatric Cardiology. Natural history and clinical outcome of "uncorrected" scimitar syndrome patients: a multicenter study of the italian society of pediatric cardiology. Rev Esp Cardiol (Engl Ed). 2013; 66(7): 556-60.

[10] Korkmaz, A.A., Yildiz, C.E., Onan, B., Guden, M., Cetin, G., Babaoglu, K. Scimitar syndrome: a complex form of anomalous pulmonary venous return, J. Card. Surg. 2011; 26 (5): 529-534.

[11] Oshima, Y., Hashimoto, I., Shimazu, C., Ichida, F. Atypical infantile form of scimitar syndrome with bronchomalacia. Interact Cardiovasc Thorac Surg. 2003; 2(03): 298-300.

[12] Winant, A.J., Cho, J., Alyafei, T.S., Lee, E.Y. Pediatric thoracic anatomic variants: what radiologists need to know. Radiol Clin 2017; 55: 677-691.

[13] Woodring, J.H., Howard, T.A., Kanga, J.F. Congenital pulmonary venolobar syndrome revisited. Radiographics. 1994; 14: 349-369.

[14] Örün, U.A., Özgür, S., Ceylan, O., Karademir, S., Şenocak, F., Yilmaz, O., Keskin, M. Horseshoe Lung and Scimitar Syndrome Associated with Congenital Hydrocephalus in an Infant: Case Report. Turkiye Klinikleri Cardiovascular Sciences. 2012; 24: 83-87.

[15] Sun, J., Zhang, S., Jiang, D., Yang, G. Scimitar syndrome with the left persistent superior vena cava. Surg Radiol Anat. 2009; 31: 307-309.

[16] Wang, C.C., Wu, E.T., Chen, S.J., Lu, F., Huang, S.C., Wang, J.K., Chang, C.I., Wu, M.H. Scimitar syndrome: Incidence, treatment and prognosis. Eur J Pediatr. 2008; 167:155-60.

[17] Jackson, N., Nokes, B.T., Sakata, K., Cummings, K., Vaszar, L. An unusual variant of scimitar syndrome predisposing to recurrent pneumonia. Respir Med Case Rep. 2019; 26: 240-243.

[18] Huddleston, C.B., Exil, V., Canter, C.E., Mendeloff, E.N. Scimitar syndrome presenting in infancy. Ann Thorac Surg. 1999; 67: 154-159.

[19] Ward, K.E., Mullins, C.E. Anomalous pulmonary venous connections, pulmonary vein stenosis, and atresia of the common pulmonary vein. In: The Science and Practice of Pediatric Cardiology, Garson A Jr, Bricker JT, Fisher DJ, Neish SR (Eds), Williams and Wilkins, Baltimore, 1998, 1431.

[20] Zagal, B., Book, S., Krasuski, R.A. Late “adult form” scimitar syndrome presenting with "infant form” complications $J$ Invasive Cardiol. 2006; 18: E82-5. 